\title{
Cutaneous toxicities of new targeted cancer therapies: must know for diagnosis, management, and patient-proxy empowerment
}

\author{
Thomas McFarlane ${ }^{1,2}$, Noor Rehman ${ }^{1}$, Katie Wang ${ }^{1}$, Jenny Lee ${ }^{1}$, Caitlin Carter ${ }^{1}$ \\ ${ }^{1}$ School of Pharmacy, Faculty of Science, University of Waterloo, Kitchener, ON, Canada; ${ }^{2}$ Odette Cancer Centre, Sunnybrook Health Sciences, \\ Toronto, ON, Canada \\ Contributions: (I) Conception and design: T McFarlane, C Carter; (II) Administrative support: None; (III) Provision of study materials or patients: \\ C Carter; (IV) Collection and assembly of data: All authors; (V) Data analysis and interpretation: T McFarlane, N Rehman, K Wang, J Lee; (VI) \\ Manuscript writing: All authors; (VII) Final approval of manuscript: All authors. \\ Correspondence to: Thomas McFarlane. Sunnybrook Health Sciences, 2075 Bayview Avenue, Toronto, ON M4N 3M5, USA. \\ Email: tom.mcfarlane@sunnybrook.ca.
}

\begin{abstract}
The world of oncology treatment is rapidly changing, and with the investigation into and utilization of molecular signaling pathways for cancer treatment, many new targeted small-molecule oral agents have been introduced as therapies, with more new drugs appearing every year. These agents, while generally considered less toxic overall than traditional chemotherapy, are not without adverse effects. The authors undertook an extensive literature search to determine the incidence, severity, and management strategies for small-molecule oral targeted agents approved by the FDA between 2013 and 2018. Dermatologic adverse effects are among the most frequently seen with many of these targeted therapies, and may include rashes, palmar-plantar dysesthesia, alopecia, secondary skin malignancies, and hair and nail changes. Rarely, more severe cutaneous toxicities are seen, such as Stevens-Johnson Syndrome and toxic epidermal necrolysis. In many cases, there is no specific management strategy suggested in the literature for these toxicities, but frequent monitoring of the skin, prophylactic management of palmar-plantar dysesthesia, use of corticosteroids and/or antihistamines, and intervention with dose interruption are suggested depending on circumstance and severity. Patient education and timely intervention is warranted in order to ensure that patient treatment is optimized.
\end{abstract}

Keywords: Targeted agents; dermatologic toxicities; cancer therapy

Submitted Mar 08, 2019. Accepted for publication Jul 31, 2019.

doi: $10.21037 /$ apm.2019.08.05

View this article at: http://dx.doi.org/10.21037/apm.2019.08.05

\section{Introduction}

The landscape of cancer treatment has drastically changed in the last two decades, with molecular targeted therapies becoming ever more prominent as therapeutic options (1). Due in part to the work of Hanahan and Weinberg in categorizing the hallmarks of malignancy and the rise in the use of cancer genomics to guide treatment selection, we are now using more of these targeted therapies than ever before $(2,3)$. Furthermore, we can expect to see increases in the number and breadth of these agents in the future, as we move toward precision medicine in oncology and more therapeutic targets are identified. In 2016, it was estimated that of 631 identified agents in the oncology pharmaceutical pipeline, $47 \%$ were targeted small molecules which are given orally (1).

Given that small molecule targeted agents tend to have a cytostatic mechanism of action and exhibit anti-growth and anti-metastatic effects rather than being cytotoxic, they have found widespread use as palliative treatments in advanced disease (4). As a result, clinicians need to be cognizant of the common adverse effects which can occur as a result of the use of these compounds. Traditionally, one of the reasons for pursuing molecular targeted therapy has been the theory that its targeted nature makes it easier 
for patients to tolerate compared to traditional cytotoxic chemotherapy. While it may be true that potentially dangerous adverse effects such as myelosuppression are not as common with targeted agents, it has become clear that use of these medications is not without risk and some patients do develop adverse effects that can lead to treatment discontinuation or delay and a significant impact on quality of life (4). This is especially important when we consider that the point of treatment with targeted therapies is often palliation of disease, and that patients with cancer are living longer lives with the incremental improvements we are seeing in efficacy as more and better therapies are introduced. Since quality of life issues should be considered front and centre to this patient group, and in light of the fact that adherence to therapy has the potential to be markedly impacted by toxicity which may in turn negatively affect disease outcomes, it is critical for clinicians to be familiar with the toxicity patterns of targeted agents in order to best inform and empower patients with respect to side effect management and to ensure treatment is optimal.

Although many different molecular targets are currently being exploited therapeutically in cancer treatment, there are a few common threads seen with this approach as far as toxicity is concerned. One very common adverse effect that has been noted with the use of many targeted agents has been dermatologic toxicity, which can present in a myriad of ways (5). One of the first classes of agents to demonstrate skin-related adverse effects was the EGFR inhibitor class, which first came to prominence as a lung cancer therapy in the mid-2000s (6). Patients taking EGFR inhibitors developed a characteristic acneiform rash of varying severity which tended to develop within the first 2 weeks of initiating treatment. In addition, alopecia, eyelash abnormalities, nail and periungual alterations, xerosis, and pruritus were often seen (6). These adverse effects and their management are well described elsewhere in the literature and are a prime example of the way in which modification of cellular signaling pathways can lead to dermatologic toxicities.

Beyond EGFR inhibitors, toxicity to the skin has been reported with various other types of molecular targeted therapies, and is one of the more common adverse effects seen with treatment (7). Our purpose in conducting this review is to outline the specific presentation of dermatologic toxicities in these recently introduced targeted therapies, specifically those with initial FDA approvals between 2013-2018; to outline the significance of these toxicities for patients receiving them for palliation of cancer; and to examine potential management strategies used to ameliorate them.

\section{Methods}

In consultation with the authors, a literature search was developed and conducted in December 2018, by a research librarian (CC) in the following databases: PubMed (MEDLINE), Ovid Embase, and Ovid International Pharmaceutical Abstracts. Where applicable, search strategies included a combination of keywords and subject headings, with all keywords restricted to the title and abstract fields only. Broad terms related to cutaneous, dermatologic, and skin toxicities were utilized, as well as specific terminology like rash, hand-foot syndrome, nail disease, and Stevens-Johnson syndrome, among many others. These search terms were combined with terminology for newer, oral cancer drugs, like PARP inhibitors, BRAF/MEK inhibitors, EML4 ALK inhibitors, CDK 4/6 inhibitors, c-MET inhibitors, and BTK inhibitors. All database searches were limited to a date range of 2013-2018. The searches generated a total of 6,771 results, which were imported into EndNote X8 software, where duplicate removal occurred. Additional, more limited searches were conducted after this to examine dermatologic toxicities with copanlisib, idelalisib, and regorafenib, as these were missed in the initial search and it was felt that they should be included in the review. These generated a total of 232 additional results. The complete search strategies can be found in Appendix 1. Medications included in the review were limited to those receiving initial FDA approval between 2013 and 2018, with the exception of BRAF inhibitors used in combination with MEK inhibitors receiving initial FDA approval in this timeframe, crizotinib as a first line ALK inhibitor, and regorafenib. All included medications are summarized in Table 1.

\section{Dermatologic toxicities of oral targeted therapies}

\section{BRAF/MEK inbibitor combinations}

The BRAF inhibitors target the mutated V600E BRAF kinase which is responsible for upregulated cellular signaling in various tumour types (44). They were originally licensed for use in advanced melanomas, which often harbour this mutation. Recently, their labeling has expanded to include BRAF V600E mutated non-small cell 
Table 1 Small molecule oral anti-cancer agents included in review of dermatologic toxicities

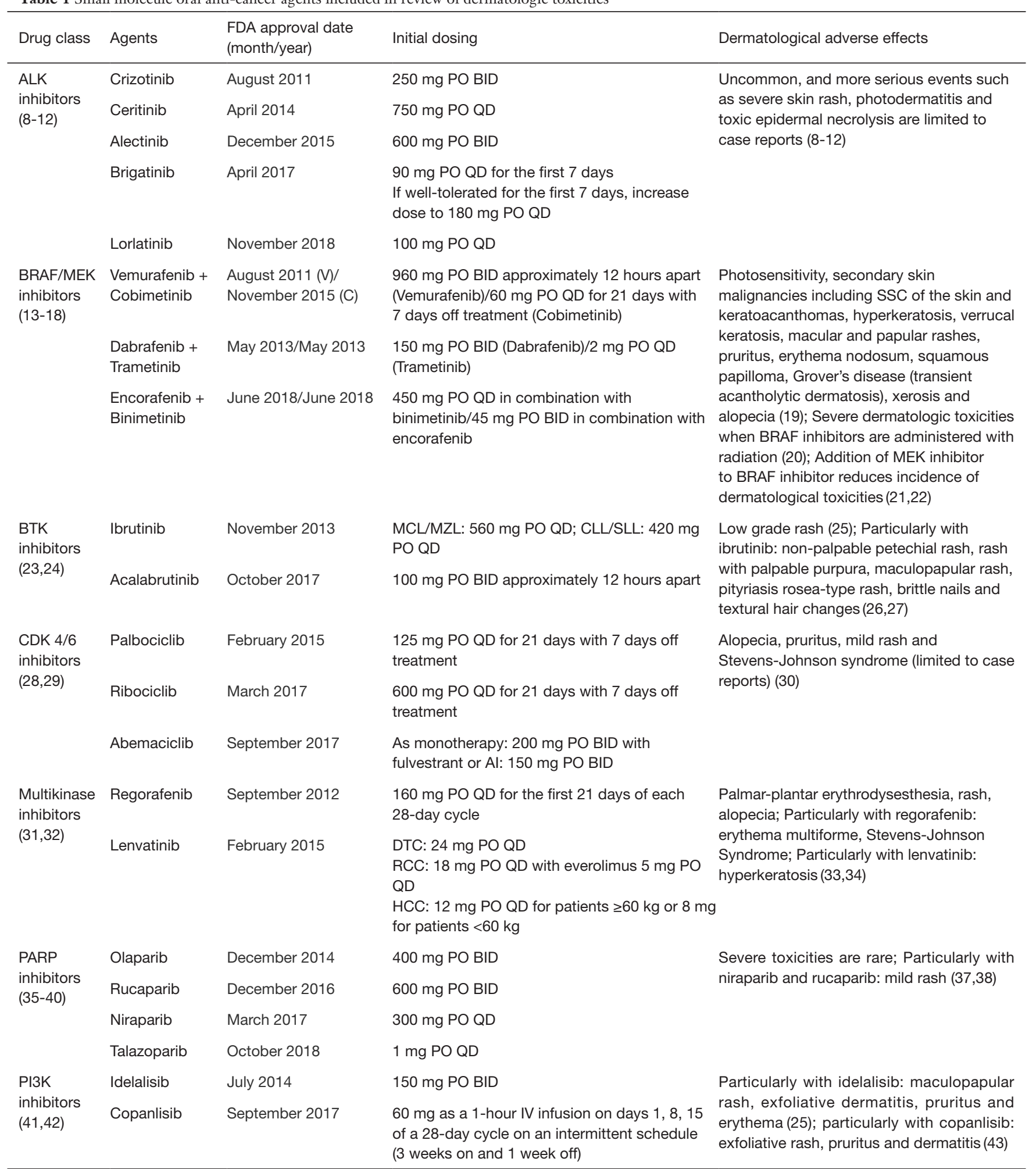

PO, orally; BID, twice daily; QD, once daily; SCC, squamous cell carcinoma; MCL, mantle cell lymphoma; MZL, marginal zone lymphoma; CLL, chronic lymphocytic leukemia; SLL, small lymphocytic lymphoma; AI, aromatase inhibitor; DTC, differentiated thyroid cancer; RCC, renal cell carcinoma; HCC, hepatocellular carcinoma; IV, intravenous. 
lung cancers (NSCLCs), thyroid cancers, and any other malignancy that tests positive for the $\mathrm{V} 600 \mathrm{E}$ mutation (13-15). Three agents targeting BRAF are currently available; these include vemurafenib, dabrafenib, and encorafenib. BRAF inhibition has been demonstrated to induce dramatic responses in mutated tumours, however these responses are relatively short-lived and malignant cells eventually develop resistance (45). Overall, BRAF inhibitors are generally well tolerated; however, a number of dermatologic toxicities have been noted which relate to blockade of the BRAF pathway (19). Patients will sometimes develop photosensitivity which can be quite profound. Use of BRAF inhibitors has also been linked to development of secondary skin malignancies, including squamous cell carcinomas (SCC) of the skin and keratoacanthomas. A myriad of other cutaneous adverse effects have been reported, including actinic keratosis, rash, erythema nodosum, squamous papillomas, Grover's disease (transient acantholytic dermatosis), panniculitis, hand-foot syndrome, and xerosis (19). Additionally, severe dermatologic toxicities have been reported when BRAF inhibitors have been administered concurrently with radiation (20). Alopecia has also been noted with BRAF inhibitors. A meta-analysis conducted by Belum et al. reported that the median incidence of alopecia with vemurafenib was $23.7 \%$ (95\% CI, 9.6-47.5\%) and with dabrafenib was $18.9 \%$ (95\% CI, $10.5-31.5 \%)(46)$.

In 2013, the first MEK inhibitor, trametinib, was licensed for use in combination with BRAF inhibitors in metastatic melanoma (16). MEK inhibitors target mitogen-activated protein kinases which leads to downregulation of MAPK/ ERK pathway signaling which is often upregulated in malignancies and act synergistically with BRAF inhibitors, as it has been demonstrated in several melanoma trials that the combination confers superior progression free survival (PFS) and overall survival (OS) over BRAF monotherapy (47). Following the approval of trametinib, two other MEK inhibitors, cobimetinib and binimetinib were also approved $(17,18)$. It has been noted that in addition to improved survival outcomes, addition of a MEK inhibitor to BRAF therapy reduces incidence of various cutaneous toxicities. In a 2013 Australian retrospective cohort study published by Carlos $e t$ $a l$., the cohort receiving the combination therapy experienced significantly less verrucal keratosis, cutaneous SCC, and Grover's disease than the cohort on monotherapy (21). Additionally, a cohort study of 44 patients performed by Sanlorenzo et al. and published in 2014 determined that the development of cutaneous adverse events was reduced (21 events vs. 8, $\mathrm{P}=0.012$ ) and the time to development was longer for the BRAF/MEK combination vs. BRAF alone, although it should be noted that this was a small retrospective trial with a limited number of patients (22). A set of management strategies for BRAF/MEK combination dermatologic toxicities has been proposed by Welsh and Corrie in a 2015 paper (48). They suggest using soap substitutes and emollients for dry skin, topical steroids and analgesia for erythema nodosum type rash, which may be stepped up to oral corticosteroids (prednisolone $0.5 \mathrm{mg} / \mathrm{kg} / \mathrm{day}$ or equivalent for 5-7 days) if necessary, and a sunscreen of greater than SPF 30 for prevention of photosensitivity. Macular/Papular rash should be treated according to NCICTCAE grading, with grade 1 rashes being observed, grade 2 rashes treated with antihistamines and emollient creams or, if necessary, topical steroids, grade 3 rashes treated as grade 2 but with drug interruption until resolution to grade 1 and consideration of oral corticosteroids as suggested above with erythema nodosum, and grade 4 rashes with discontinuation of therapy and hospitalization if warranted.

Consensus guidelines have been published by the Eastern Cooperative Oncology Group (ECOG) on avoiding severe toxicity seen with the combination of BRAF inhibitors and radiation (20). Almost all grade 3 dermatitis reactions seen in this setting have been during concurrent radiation, and the authors suggest holding BRAF and/or MEK inhibitors for at least 3 days before and after fractionated radiation therapy (RT) and at least 1 day before and after stereotactic radiosurgery (SRS), with time off systemic therapy being minimized as much as possible. Recommendations for RT include considering a dose per fraction of less than 4 Gy unless using a stereotactic approach or the patient has a very poor prognosis or performance status.

\section{Anaplastic lymphoma kinase (ALK) inhibitors}

ALK inhibitors target the EML4-ALK fusion gene, a mutated gene which occurs in approximately $5 \%$ of lung adenocarcinomas, primarily in non-smokers (49). These agents are generally given as monotherapy in metastatic NSCLC in patients who test positive for EML4-ALK (50). The first agent targeting this fusion gene was crizotinib, with second-generation (ceritinib, brigatinib, and alectinib) and third-generation (lorlatinib) agents subsequently introduced to address the problem of developed resistance to ALK inhibition. These drugs tend to have significant efficacy in the ALK positive patients, and indeed crizotinib was initially FDA approved for this population based on 
phase I clinical trial data (51). There are some cutaneous toxicities that can result from ALK inhibitor use; however, these are uncommon and more serious events tend to be limited to case reports, including severe skin rash, erythema multiforme, photodermatitis, and toxic epidermal necrolysis cases found in our literature search (52-55). In the PROFILE 1014 phase III study of crizotinib, rash was noted in $11 \%$ of participants on the crizotinib arm, with all incidences being either grade 1 or 2 (8). Similarly, some incidence of grade 1 or 2 rash was noted in the alectinib, brigatinib, ceritinib, and lorlatinib phase II and III trials $(20 \%, 18 \%, 20 \%$, and $14 \%$, respectively) with two reports of grade 3 or higher rash in the brigatinib and ceritinib trials and one report in the lorlatinib trial (9-12). We could not find any specific management strategy for ALK inhibitor-related rash in the literature.

\section{Cyclin-dependent kinase (CDK) 4/6 inhibitors}

CDK 4/6 inhibitors are agents which target CDKs, a group of enzymes which promote cell division and proliferation and can be upregulated in malignant cells (56). The mechanism of action of these agents involves blockade of the ability of the CDK 4/6 and cyclin D1 protein complex from phosphorylating the retinoblastoma (RB) protein, thus inducing cell cycle arrest at G1. Currently, CDK 4/6 inhibitors are indicated in metastatic breast cancer in hormone-receptor positive (HR+) and HER2 negative patients and are primarily used in combination with estrogen blockade (28). Three agents are currently on the market; palbociclib, ribociclib, and abemaciclib. Abemaciclib, additionally, is indicated as monotherapy by the FDA after failure of estrogen inhibition and chemotherapy (29). Of the dermatologic adverse events seen with this class of agents, the most noteworthy is alopecia, which was seen in the PALOMA-2 trial comparing the combination of palbociclib and letrozole with letrozole alone, at a rate of $32.9 \%$ for grades 1 and 2 compared with $15.8 \%$ in the letrozole alone arm (57). High incidences of grade 1 and 2 alopecia were also seen in MONARCH-3 with abemaciclib and in MONALEESA-2 with ribociclib $(58,59)$. There is no specific evidence-based management strategy for prevention or treatment of alopecia with these agents, but patients should be cautioned that they may experience it. As with the ALK inhibitors above, mild rash has been noted in some patients utilizing CDK 4/6 inhibitors. Rash with the use of these agents is rarely severe, though case reports of Stevens-Johnson syndrome have been noted in the literature (30).

\section{Poly (ADP-Ribose) polymerase (PARP) inhibitors}

PARP inhibitors are agents which inhibit poly (ADP-ribose) polymerase, a group of enzymes involved in cellular DNA repair, and have been shown to be effective in malignancies which harbour a deficiency in repair of DNA damage, including those tumours which are BRCA mutationassociated (60). Inhibition of PARP in these tumours leads to an inability to repair DNA damage because of a defect in the homologous repair pathway which ordinarily would repair double strand DNA breaks, a concept known as synthetic lethality where loss of both the BRCA and PARP pathways leads to cell death. Currently marketed PARP inhibitors include olaparib, rucaparib, niraparib, and talazoparib, and indications for these agents include use in BRCA mutated ovarian and breast cancer $(35,36)$.

Severe dermatologic toxicities with PARP inhibitors are not common; however, similar to the other targeted agents so far covered here, mild rashes related to treatment do occur, particularly with niraparib and rucaparib. Incidence of grade 1 or 2 rash reported in phase III clinical trials in the FDA labeling was $21 \%$ with niraparib and $43 \%$ with rucaparib $(37,38)$. Approximately $5 \%$ of patients experienced a similar rash with olaparib in the OlympiAD trial, and there was no rash reported with talazoparib in the EMBRACA trial in breast cancer patients $(39,40)$. Cases of grade 3 and 4 rash were rare in these trials, with incidences of $1 \%$ and $0.5 \%$ with niraparib and rucaparib, respectively, and no cases reported with the other agents.

\section{Phosphoinositide 3-kinase (PI3K) inhibitors}

PI3K inhibitors act to inhibit a group of enzymes which is part of the PI3K/Akt/mammalian target of rapamycin (mTOR) signaling axis which is often upregulated in cancer cells and leads to cell proliferation via the nuclear factor kappa B pathway (61). There are two PI3K inhibitors approved for clinical use today, idelalisib and copanlisib, with several more agents currently undergoing clinical trials. Idelalisib is indicated for use in relapsed or refractory chronic lymphocytic leukemia (CLL), follicular lymphoma (FL), and small lymphocytic leukemia (SLL), and copanlisib for relapsed FL after two previous failed lines of therapy $(41,42)$.

With idelalisib, a maculopapular rash is sometimes seen, which can be severe in rare cases. Rash of any grade 
was noted in $10-22 \%$ of patients in clinical trials, but severe (grade 3 or 4 ) rashes were only seen in $0-2 \%(52)$. One case of toxic epidermal necrolysis was noted in combination with rituximab and bendamustine (61). There is no specific management strategy suggested for this rash other than symptomatic treatment and, in severe cases, dose interruption or discontinuation. There is no current literature available on the utility of corticosteroids or antihistamines in this setting (25).

Severe dermatologic toxicities with copanlisib are well documented, and although rare can include maculopapular rash, exfoliative rash, pruritis, and dermatitis (43). The drug's FDA labeling reports a combined incidence of severe dermatologic toxicities of $3.4 \%$ and suggests withholding the drug until resolved if grade 3 toxicity is noted, then reintroducing at a reduced dose from 60 to $45 \mathrm{mg}$ or 45 to $30 \mathrm{mg}$ once resolution occurs (42).

\section{Bruton's tyrosine kinase (BTK) inhibitors}

BTK is a protein which is responsible for mediating oncogenic signaling and survival of leukemic cells in several different types of B-cell malignancies. Overactivity of BTK alters cell chemotaxis and facilitates the development of a supportive microenvironment for survival and proliferation of malignant B-cells (62). This has led to development of BTK inhibitors, which induces apoptosis in various B-cell lines. The first agent in this class to be introduced was ibrutinib, with a second agent, acalabrutinib, being subsequently approved $(23,24)$. BTK inhibitors are indicated for use in mantle cell lymphoma (MCL), CLL/ SLL, marginal zone lymphoma, and FL.

Ibrutinib related rash occurs relatively frequently, with a 2015 systematic review determining an incidence of $15-28 \%$ of all grade rash (63). A wide presentation of rash types has been reported, including non-palpable petechial rashes, rashes with palpable purpura, maculopapular rash, and pityriasis rosea-type rash (25). Rashes are generally grade 1 or 2 and will often spontaneously resolve without the need for treatment, but there have been cases reported of more severe rash for which dose interruption was required. Dose reduction or discontinuation has not been reported. No specific rash management strategy exists in the literature related to ibrutinib. There have also been reports of ibrutinib-induced nail and hair toxicity, which are quite commonly seen. Bitar et al. conducted a prospective study to determine the incidence of hair and nail changes in a cohort of 66 patients receiving ibrutinib therapy for CLL, which found that brittle fingernails were noted in 44 patients $(67 \%)$ and brittle toenails were seen in 15 patients (23\%) (26). These tended to appear several months after starting ibrutinib therapy. It is thought that the pathogenesis of nail changes with ibrutinib is due to disruption of disulfide bonds between cysteine residues, which leads to a breakdown in the integrity of keratinassociated fibrous proteins making up the nail plate (27). Treatment of these changes is not generally considered necessary, although it is recommended that patients keep nails short. Clear nail polish can be used for cosmetic effect, and there is anecdotal evidence that biotin $2.5 \mathrm{mg}$ once daily may be effective, although this needs to be validated. Textural hair changes were also noted in the Bitar study in 17 patients $(26 \%)$ and were characterized as a softening and straightening, although there have also been reports of increased curliness (26).

Acalabrutinib is a newer agent, and thus there is a paucity of clinical experience with it and little in the literature regarding dermatologic toxicity. Rash has been noted with it in clinical trials, though similar to ibrutinib, this rash was generally low grade (18\% grade 1 or 2 rash in the LY-004 trial) and severe rash was rare (0.8\%) (24).

\section{Multikinase inhibitors}

There are many multikinase inhibitors in use in oncology, however most of them did not fall within the specified date range of initial approvals we examined. Lenvatinib, however, is a small-molecule oral inhibitor of the vascular endothelial growth factor receptors 1, 2, and 3 (VEGFR1, VEGFR2, VEGFR3). It has also been demonstrated to inhibit other receptor tyrosine kinases related to tumour growth and metastases, including RET, KIT, fibroblast growth factor receptors (FGFR) 1, 2, 3, and 4, and platelet-derived growth factor receptor alpha (PDGFR $\alpha$ ) (33). Lenvatinib is indicated clinically for treatment of locally recurrent or metastatic radioactive iodine-refractory differentiated thyroid cancer (DTC), in combination with everolimus for metastatic renal-cell carcinoma, and as a first-line agent for hepatocellular carcinoma (31). There are various cutaneous toxicities that have been seen with lenvatinib, including rash, palmar-plantar erythrodysesthesia (PPE), alopecia, and hyperkeratosis.

PPE, or hand-foot syndrome, is characterized by tender, inflamed, erythematous lesions affecting the palms of the hands and the soles of the feet, and is a specific concern with lenvatinib. Tingling and paresthesias are 
often experienced along with the redness and swelling. In the Phase III SELECT study examining lenvatinib use in thyroid cancer, $31.8 \%$ of patients experienced PPE, however this was largely grade 1 or 2 , with only $3.3 \%$ of patients experiencing grade 3 or worse toxicity (34). Thus, lenvatinib dose can generally be continued if PPE occurs, although patients should be cautioned about what to look for and use prophylactic strategies for prevention, including liberal use of moisturizer and avoiding sources of friction or heat on palms or soles. Takahashi et al. published a proposed management strategy for PPE related to lenvatinib which involves continuing at full dose and using moisturizing cream for grade 1 presentation, and interrupting dose and use of betamethasone valerate topically for grade 2 or 3 presentation until resolution to grade 0 or 1 (33). Dose reductions upon resolution are recommended in this strategy in the case of grade 3 presentation or rapid (within 1 week after initiating) appearance of grade 2 PPE. Dosage can be reduced in a stepwise manner from the full dose of 24 to 20,14 , and $10 \mathrm{mg}(31)$.

Rash occurred in the SELECT trial at a rate of $21.4 \%$ and was almost entirely grade 1 or 2 . Alopecia was seen in $12 \%$ of patients and hyperkeratosis in $7 \%$ and there was no grade 3 or higher toxicity seen with either adverse effect. There is no specific published management strategy suggested for any of these toxicities, but patients should be made aware that they could happen.

Regorafenib, another multi-kinase inhibitor that inhibits VEGFR1-3, TIE2, KIT, RET, PDGFR, and FGFR, is becoming more commonly used, particularly in colorectal cancer, hepatocellular carcinoma, and gastrointestinal stromal tumours (GIST) (32). As with lenvatinib, several presentations of dermatologic adverse effects can result from regorafenib use. The most common of these is PPE, which was found to occur in $61 \%$ of patients overall in a meta-analysis published in 2013, and $20 \%$ of PPE was noted to be grade 3 (64). A review of practical advice on regorafenib use published by McLellan et al. in 2015 suggested prophylactic measures such as avoiding heat and constrictive footwear as well as vigorous activities placing stress on the extremities, liberal use of moisturizer, and wearing of thick cotton gloves and socks to protect hands and feet (65). A full skin exam should be conducted prior to initiation of therapy to ensure that changes can be easily detected. Interventions for grade 1 symptoms in this review included use of keratolytics, with $10-40 \%$ urea or $5-10 \%$ salicylic acid being suggested, as well as topical analgesics such as lidocaine gel and skin cooling packs for pain.
Clobetasol $0.05 \%$ ointment can be added for erythema if grade 2 symptoms develop, and topical antibiotics or antiseptics for desquamation in the case of grade 3 symptoms. Dose reduction from the standard $160 \mathrm{mg}$ daily 21 days out of 28 was suggested at grade 2, and interruption of treatment for at least 7 days until resolution of toxicity at grade 3 (or unresolved grade 2), followed by reintroduction at reduced dosage levels of 120 and $80 \mathrm{mg}$ daily. In patients developing hyperkeratotic lesions, tazarotene $0.1 \%$ topically as an antiproliferative agent may be helpful. Interestingly, a Japanese group published a retrospective study in 2018 suggesting that PPE severity may be associated with improved efficacy in metastatic colorectal cancer (66).

Maculopapular rash can also occur with regorafenib treatment with an incidence of $18-26 \%$ in the literature, although most rashes are grade 1 or $2(67,68)$. Preventative measures are again suggested, including alcohol-free emollients, mild soaps, and avoiding direct sun exposure, and all grades of rash can be effectively managed with antihistamines and a short course of clobetasol propionate $0.05 \%$ in cold cream (69). Severe, generalized toxicities such as Stevens-Johnson syndrome are rare, but there have been case reports published in the literature $(70,71)$.

\section{Discussion}

Clearly, when dealing with oral molecular targeted agents there is a wide range of potential dermatologic adverse events that may occur, ranging from mild grade 1 rash to severe and potentially life threatening conditions such as toxic epidermal necrolysis. Vigilance by the patient, therefore, is extremely important, as they will be the first to notice if something is amiss. Educating the patient on what to look for and expect in terms of skin toxicity with a given targeted agent should be part of the teaching process upon initiation of treatment. Prophylactic strategies such as frequent moisturizing, use of mild cleansers, and avoidance of skin irritants are appropriate and depending on the circumstance and drug used may help prevent problems from occurring.

Adherence to therapy is extremely important to optimize outcomes in palliative cancer therapy. There is generally a lack of published evidence on the subject of adherence to TKIs in areas other than BCR-ABL inhibitor therapy in chronic myelogenous leukemia (CML). The CML population demonstrates that, in addition to a clear link between outcomes and degree of adherence to therapy, toxicity had a direct impact on adherence in multiple assessments, with $17.6 \%$ 
of patients in one survey admitting to skipping doses directly due to side effects (72). A 2015 study by Zuo et al. on dermatologic toxicity with cabozantinib, which causes PPE, hair pigmentation, xerosis, scrotal erythema and ulceration, and nail splinter hemorrhages, concluded that early detection and prompt treatment of toxicities may improve adherence to therapy (73). Thus, it is very important for us as clinicians to teach and empower patients to prophylactically manage toxicities and report them promptly, and intervene on an expedient basis when necessary. Rash in particular is an adverse effect which is likely under-recognized and undertreated, and a discussion with the patient should be had around how a rash may impact quality of life and how rashes may be managed appropriately. Quality of life in and of itself should be considered one of the primary goals of therapy in palliative cancer treatment, and data exists in the population of patients using EGFR inhibitors regarding impact of dermatologic toxicities on quality of life that can likely be extrapolated to the new TKIs. Yang et al. published a prospective study of 344 EGFR mutation-positive lung cancer patients utilizing EuroQol five-dimension and World Health Organization Quality-of-Life-brief questionnaires in order to determine scores for patients taking erlotinib, gefitinib, and afatinib (74). They determined that quality of life scores appeared to be worse in patients taking afatinib, and that one of the determinants of this was impact of treatment on body image, with dermatologic toxicities such as increased rates of paronychia and folliculitis contributing to this. Thus, clinicians need to be cognizant of the fact that dermatologic manifestations seen with these agents can be burdensome for patients and that they should be treated seriously.

\section{Conclusions}

Dermatologic toxicities are not uncommon with new targeted agents, with secondary cutaneous malignancies being seen with BRAF inhibitors, alopecia with CDK 4/6 inhibitors, hair and nail toxicity with ibrutinib, and handfoot syndrome seen with lenvatinib, in addition to other, more rare toxicities. Low grade rash is very common and tends to be seen across targeted therapies. In order to properly empower the patient and manage treatment optimally, it is important to ensure that patients are well informed of the possibility and presentation of these toxicities, how to prophylactically manage them, and when to report to a health care provider. Prescribers should be aware of the management strategies that exist to treat these toxicities and intervene on an expedient basis when necessary to improve treatment outcomes and preserve patient quality of life in the palliative setting.

\section{Acknowledgments}

Funding: None.

\section{Footnote}

Provenance and Peer Review: This article was commissioned by the Guest Editors (Jan Gaertner, Charles B. Simone II and Fiona Lim) for the series "Clinical Challenges and Pitfalls in Early Palliative Care: Practical Knowledge and Guidance from other Medical Specialties" published in Annals of Palliative Medicine. The article was sent for external peer review organized by the Guest Editors and the editorial office.

Conflicts of Interest: All authors have completed the ICMJE uniform disclosure form (available at http://dx.doi. org/10.21037/apm.2019.08.05). The series "Clinical Challenges and Pitfalls in Early Palliative Care: Practical Knowledge and Guidance from other Medical Specialties" was commissioned by the editorial office without any funding or sponsorship. Dr. McFarlane has received honoraria as a consultant from AstraZeneca to this section. The authors have no other conflicts of interest to declare.

Ethical Statement: The authors are accountable for all aspects of the work in ensuring that questions related to the accuracy or integrity of any part of the work are appropriately investigated and resolved.

Open Access Statement: This is an Open Access article distributed in accordance with the Creative Commons Attribution-NonCommercial-NoDerivs 4.0 International License (CC BY-NC-ND 4.0), which permits the noncommercial replication and distribution of the article with the strict proviso that no changes or edits are made and the original work is properly cited (including links to both the formal publication through the relevant DOI and the license). See: https://creativecommons.org/licenses/by-nc-nd/4.0/.

\section{References}

1. Global Oncology Trends 2017. Available online: 
https:/www.communityoncology.org/wp-content/ uploads/2017/06/QIIHI_Oncology_Trend_

Report_2017_Advances_Complexity_Cost.pdf. Accessed February 28, 2019.

2. Hanahan D, Weinberg RA. The Hallmarks of Cancer. Cell 2000;100:57-70.

3. Hanahan D, Weinberg RA. Hallmarks of Cancer: The Next Generation. Cell 2011;144:646-74.

4. Targeted Cancer Therapies Fact Sheet, National Cancer Institute. Available online: https://www.cancer.gov/aboutcancer/treatment/types/targeted-therapies/targetedtherapies-fact-sheet. Accessed February 28, 2019.

5. Kao S, Friedmen A. Supportive Oncodermatology: Addressing dermatologic adverse events associated with oncologic therapies. Oncology Issues 2018;33:64-75.

6. Lynch TJ Jr, Kim ES, Eaby B, et al. Epidermal growth factor receptor inhibitor-associated cutaneous toxicities: an evolving paradigm in clinical management. Oncologist 2007;12:610-21.

7. Kao PH, Chen JS, Chung WH, et al. Cutaneous Adverse Events of Targeted Anticancer Therapy: A Review of Common Clinical Manifestations and Management. J Cancer Res Pract 2015;2:271-84.

8. Solomon BJ, Mok T, Kim DW et al. First-Line Crizotinib versus Chemotherapy in ALK-Positive Lung Cancer. N Engl J Med 2014;371:2167-77.

9. ALECENSA® [Package Insert]. South San Francisco, CA: Genentech Inc., 2017.

10. ALUNBRIG ${ }^{\mathrm{TM}}$ [Package Insert]. Cambridge, MA: ARIAD Pharmaceuticals Inc., 2017.

11. ZYKADIA ${ }^{\mathrm{TM}}$ [Package Insert]. East Hanover, NJ: Novartis Pharmaceuticals Corp., 2017.

12. LORBRENA® [Package Insert]. New York, NY: Pfizer Labs, 2018.

13. ZELBORAF® [Package Insert]. South San Francisco, CA: Genentech, Inc, 2017.

14. TAFINLAR® [Package Insert]. East Hanover, NJ: Novartis Pharmaceuticals Corp., 2017.

15. BRAFTOVI® [Package Insert]. Boulder, CO: Array BioPharma Inc., 2018.

16. MEKINIST® [Package Insert]. East Hanover, NJ: Novartis Pharmaceuticals Corp., 2018.

17. COTELLIC® [Package Insert]. South San Francisco, CA: Genentech, Inc., 2018.

18. MEKTOVI® [Package Insert]. Boulder, CO: Array BioPharma Inc., 2018.

19. Gençler B, Gönül M. Cutaneous Side Effects of BRAF Inhibitors in Advanced Melanoma: Review of the Literature. Dermatol Res Pract 2016. doi: $10.1155 / 2016 / 5361569$.

20. Anker CJ, Grossmann KF, Atkins MB et al. Avoiding Severe Toxicity From Combined BRAF Inhibitor and Radiation Treatment: Consensus Guidelines from the Eastern Cooperative Oncology Group (ECOG). Int J Radiat Oncol Biol Phys 2016;95:632-46.

21. Carlos G, Anforth R, Clements A, et al. Cutaneous Toxic Effects of BRAF Inhibitors Alone and in Combination With MEK Inhibitors for Metastatic Melanoma. JAMA Dermatol 2015;151:1103-09.

22. Sanlorenzo M, Choudhry A, Vujic I, et al. Comparative profile of cutaneous adverse events: BRAF/MEK inhibitor combination therapy versus BRAF monotherapy in melanoma. J Am Acad Dermatol 2014;71:1102-9.

23. IMBRUVICA® [Package Insert]. Sunnyvale, CA: Pharmacyclics LLC, 2017.

24. CALQUENCE® [Package Insert]. Wilmington DE: AstraZeneca Pharmaceuticals LP, 2017.

25. de Weerdt I, Koopmans SM, Kater AP, et al. Incidence and management of toxicity associated with ibrutinib and idelalisib: a practical approach. Haematologica 2017;102:1629-39.

26. Bitar C, Farooqui MZ, Valdez J, et al. Hair and Nail Changes During Long-term Therapy With Ibrutinib for Chronic Lymphocytic Leukemia. JAMA Dermatol 2016;152:698-701.

27. Manica LA, Cohen PR. Ibrutinib-Associated Nail Plate Abnormalities: Case Reports and Review. Drug Saf Case Rep 2017;4:15.

28. National Comprehensive Cancer Network. Non SmallCell Lung Cancer (Version 3.2019). Available online: https://www.nccn.org/professionals/physician_gls/pdf/ nscl.pdf. Accessed March 1, 2019.

29. VERZENIO ${ }^{\mathrm{TM}}$ [Package Insert]. Indianapolis, IN: Lilly USA, LLC, 2018.

30. Karagounis T, Vallurupalli M, Nathan N, et al. StevensJohnson syndrome-like eruption from palbociclib in a patient with metastatic breast cancer. JAAD Case Rep 2018;4:452-4

31. LENVIMA ${ }^{\mathrm{TM}}$ [Package Insert]. Woodcliff Lake, NJ: Eisai, Inc., 2018.

32. STIVARGA® [Package Insert]. Wayne, NJ: Bayer HealthCare Pharmaceuticals., 2017.

33. Takahashi S, Kyota N, Tahara M. Optimal use of lenvatinib in the treatment of advanced thyroid cancer. Cancers of the Head and Neck 2017;2:7.

34. Schlumberger M, Tahara M, Wirth LJ, et al. Lenvatinib 
versus placebo in radioiodine-refractory thyroid cancer. $\mathrm{N}$ Engl J Med 2015;372:621-30.

35. Mirza MR, Pignata S, Ledermann JA. Latest clinical evidence and further development of PARP inhibitors in ovarian cancer. Ann Oncol 2018;29:1366-76.

36. Food and Drug Administration. Available online: https://www.fda.gov/Drugs/InformationOnDrugs/ ApprovedDrugs/ucm623540.htm. Accessed March 1, 2019.

37. Mirza MR, Monk BJ, Herrstedt J, et al. Niraparib Maintenance Therapy in Platinum-Sensitive, Recurrent Ovarian Cancer. N Engl J Med 2016;375:2154-64.

38. Coleman RL, Oza AM, Lorusso D, et al. Rucaparib maintenance treatment for recurrent ovarian carcinoma after response to platinum therapy (ARIEL3): a randomised, double-blind, placebo-controlled, phase 3 trial. Lancet 2017;390:1949-61.

39. Robson ME, Im SA, Senkus E et al. Olaparib for Metastatic Breast Cancer in Patients with a Germline BRCA Mutation. N Engl J Med 2017;377:523-33.

40. Litton JK, Rugo HS, Ettl J, et al. Talazoparib in Patients with Advanced Breast Cancer and a Germline BRCA Mutation. N Engl J Med 2018;379:753-63.

41. ZYDELIG® [Package Insert]. Foster City, CA: Gilead Pharmaceuticals, 2017.

42. ALIQOPA ${ }^{\mathrm{TM}}[$ Package Insert]. Whippany, NJ: Bayer HealthCare Pharmaceuticals, 2017.

43. Mensah FA, Blaize JP, Locke BJ. Spotlight on copanlisib and its potential in the treatment of relapsed/refractory follicular lymphoma: evidence to date. Onco Targets Ther 2018;11:4817-27.

44. Sharma A, Shah SR, Illum H, et al. Vemurafenib: targeted inhibition of mutated BRAF for treatment of advanced melanoma and its potential in other malignancies. Drugs 2012;72:2207-22.

45. Arozarena I, Wellbrock C. Overcoming Resistance to BRAF Inhibitors. Ann Transl Med 2017;5:387.

46. Belum VR, Marulanda K, Ensslin C et al. Alopecia in patients treated with molecularly targeted anticancer therapies. Ann Oncol 2015;26:2496-502.

47. Luke JJ, Ott PA, Shapiro GI. The biology and clinical development of MEK inhibitors for cancer. Drugs 2014;74:2111-28.

48. Welsh SJ, Corrie PG. Management of BRAF and MEK inhibitor toxicities in patients with metastatic melanoma. Ther Adv Med Oncol 2015;7:122-36.

49. Ardini E, Galvani A. ALK Inhibitors, a Pharmaceutical Perspective. Front Oncol 2012;2:17.
50. National Comprehensive Cancer Network. Breast Cancer (Version 4.2018). Available online: https://www.nccn. org/professionals/physician_gls/pdf/breast.pdf. Accessed March 1, 2019.

51. Kazandjian D, Blumenthal GM, Chen H, et al. FDA Approval Summary: Crizotinib for the Treatment of Metastatic Non-Small Cell Lung Cancer With Anaplastic Lymphoma Kinase Rearrangements. Oncologist 2014;19:e5-11.

52. Yang S, Wu L, Li X, et al. Crizotinib-associated toxic epidermal necrolysis in an ALK-positive advanced NSCLC patient. Mol Clin Oncol 2018;8:457-9.

53. Oser MG, Jänne PA. A Severe Photosensitivity Dermatitis Caused by Crizotinib. J Thorac Oncol 2014;9:e51-3.

54. Zheng SY, Shen W, Peng YM, et al. Treatment of severe rash caused by crizotinib with both traditional Chinese medicine and Western medicine. Medicine 2018;97:e13088.

55. Kimura T, Sowa-Osako J, Nakai T, et al. Alectinib-Induced Erythema Multiforme and Successful Rechallenge with Alectinib in a Patient with Anaplastic Lymphoma KinaseRearranged Lung Cancer. Case Rep Oncol 2016;9:826-32.

56. Pernas S, Tolaney SM, Winer EP, et al. CDK4/6 inhibition in breast cancer: current practice and future directions. Ther Adv Med Oncol 2018;10:1758835918786451.

57. Finn RS, Martin M, Rugo HS, et al. Palbociclib and Letrozole in Advanced Breast Cancer. N Engl J Med 2016;375:1925-36.

58. Goetz MP, Toi M, Campone M, et al. MONARCH 3: Abemaciclib As Initial Therapy for Advanced Breast Cancer. J Clin Oncol 2017;35:3638-46.

59. Hortobagyi GN, Stemmer SM, Burris HA, et al. Updated results from MONALEESA-2, a phase III trial of firstline ribociclib plus letrozole versus placebo plus letrozole in hormone receptor-positive, HER2-negative advanced breast cancer. Ann Oncol 2018;29:1541-7.

60. Pommier Y, O'Connor J, de Bono JS. Laying a trap to kill cancer cells: PARP inhibitors and their mechanisms of action. Sci Transl Med 2016;362:362ps17.

61. Greenwell IB, Ip A, Cohen JB. PI3K Inhibitors: Understanding Toxicity Mechanisms and Management. Oncology (Williston Park) 2017;31:821-28.

62. Seiler T, Dreyling M. Bruton's tyrosine kinase inhibitors in B-cell lymphoma: current experience and future perspectives. Expert Opin Investig Drugs 2017;26:909-15.

63. Hou Z, Belum VR, Palomba ML, et al. Systematic review of rash in cancer patients receiving ibrutinib and idelalisib. J Clin Oncol 2017;33:e12655. 
64. Belum VR, Wu S, Lacouture ME. Risk of hand-foot skin reaction with the novel multikinase inhibitor regorafenib: a meta-analysis. Invest New Drugs 2013;31:1078-86.

65. McLellan B, Ciardello F, Lacouture ME, et al. Regorafenib-associated hand-foot reaction: practical advice on diagnosis, prevention, and management. Ann Oncol 2015;26:2017-26.

66. Kobayashi K, Kawakami K, Yokokawa T, et al. Association of hand-foot skin reaction with regorafenib efficacy in the treatment of metastatic colorectal cancer. Oncology 2019;96:200-6.

67. Grothey A, Van Cutsem E, Sobrero A, et al. Regorafenib monotherapy for previously treated metastatic colorectal cancer (CORRECT): an international, multicenter, randomized, phase 3 trial. Lancet 2013;381:303-12.

68. Demitri GD, Reichardt P, Yang YK, et al. Efficacy and safety of regorafenib for advanced gastrointestinal stromal tumours after failure of imatinib and sunitinib (GRID): an international, multicenter, randomized, placebocontrolled, phase 3 trial. Lancet 2013;381:295-302.

69. De Wit M, Boers-Doets CB, Saettini A, et al. Prevention

Cite this article as: McFarlane T, Rehman N, Wang K, Lee J, Carter C. Cutaneous toxicities of new targeted cancer therapies: must knows for diagnosis, management, and patientproxy empowerment. Ann Palliat Med 2020;9(3):1296-1306. doi: 10.21037/apm.2019.08.05 and management of adverse events related to regorafenib. Support Care Cancer 2014;22:837-46.

70. Mihara Y, Yamaguchi K, Nakama T, et al. Stevens-Johnson syndrome induced by regorafenib in a patient with progressive recurrent rectal carcinoma. Gan To Kagaku Ryoho 2015;42:233-6.

71. Mii Y, Fukuoka E, Murata K, et al. A case of erythema multiforme induced by regorafenib therapy for metastatic colon cancer. Gan To Kagaku Ryoho 2014;41:1841-3.

72. Anderson KR, Chambers CR, Lam M, et al. Medication adherence among adults prescribed imatinib, dasatinib, or nilotinib for the treatment of chronic myeloid leukemia. J Oncol Pharm Pract 2014;21:19-25.

73. Zuo RC, Apolo AB, Digiovanna JJ, et al. Cutaneous adverse effects associated with the tyrosine-kinase inhibitor cabozantinib. JAMA Dermatol 2015;151:170-7.

74. Yang SC, Lin CC, Lai WW, et al. Dynamic changes in quality of life after three first-line therapies for EGFR mutation-positive advanced non-small-cell lung cancer. Ther Adv Med Oncol 2018;10:1-11. 


\section{Search strategies: PubMed (MEDLINE)}

(skin diseases[mesh] OR skin manifestations[mesh] OR drug eruptions[mesh] OR hypersensitivity[mesh] OR purpura[mesh] OR cutaneous reaction*[tiab] OR cutaneous toxicit*[tiab] OR cutaneous complication*[tiab] OR cutaneous drug eruption*[tiab] OR cutaneous adverse effect*[tiab] OR cutaneous adverse event*[tiab] OR cutaneous side effect*[tiab] OR cutaneous effect*[tiab] OR cutaneous disease*[tiab] OR cutaneous manifestation*[tiab] OR cutaneous injur*[tiab] OR cutaneous abnormalit*[tiab] OR dermatologic reaction*[tiab] OR dermatologic toxicit*[tiab] OR dermatologic complication*[tiab] OR dermatologic drug eruption*[tiab] OR dermatologic adverse effect*[tiab] OR dermatologic adverse event*[tiab] OR dermatologic side effect*[tiab] OR dermatologic effect ${ }^{*}[$ tiab] OR dermatologic disease*[tiab] OR dermatologic manifestation*[tiab] OR dermatologic injur*[tiab] OR dermatologic abnormalit*[tiab] OR skin reaction*[tiab] OR skin toxicit*[tiab] OR skin complication*[tiab] OR skin eruption*[tiab] OR skin side effect*[tiab] OR skin effect*[tiab] OR skin manifestation*[tiab] OR skin injur*[tiab] OR skin abnormalit*[tiab] OR "skin damage"[tiab] OR rash[tiab] OR rashes[tiab] OR exanthema[tiab] OR hyperpigmentation[tiab] OR hypermelanos*[tiab] OR "hand-foot syndrome”[tiab] OR "palmar-plantar erythrodysesthesia"[tiab] OR "palmoplantar erythrodysesthesia”[tiab] OR hypersensitivit*[tiab] OR allergic reaction*[tiab] OR mucocutaneous[tiab] OR nail dystroph*[tiab] OR nail disease*[tiab] OR nail disorder*[tiab] OR nail toxicit*[tiab] OR nail change*[tiab] OR petechiae[tiab] OR purpura[tiab] OR photosensiti*[tiab] OR photodermatitis[tiab] OR pruritus[tiab] OR urticarial[tiab] OR stomatit*[tiab] OR "erythema multiforme"[tiab] OR "dry skin"[tiab] OR "cutaneous lupus erythematosus" [tiab] OR dermatitis[tiab] OR dermatos*[tiab] OR erythema[tiab] OR "stevens-johnson syndrome"[tiab]) AND (poly ADP-ribose polymerase inhibitors[mesh] OR talazoparib[nm] OR olaparib[nm] OR rucaparib[nm] OR niraparib[nm] OR encorafenib[nm] OR MEK162[nm] OR vemurafenib[nm] OR GDC-0973[nm] OR dabrafenib[nm] OR trametinib[nm] OR CH5424802[nm] OR ceritinib[nm] OR crizotinib[nm] OR AP26113[nm] OR palbociclib[nm] OR ribociclib[nm] OR cabozantinib[nm] OR PCI 32765[nm] OR PARP inhibitor*[tiab] OR polyadenosine ribose polymerase inhibitor*[tiab] OR poly ADP ribose polymerase inhibitor*[tiab] OR poly ADP-ribosylation inhibitor*[tiab] OR talazoparib[tiab] OR Talzenna[tiab] OR olaparib[tiab] OR Lynparza[tiab] OR rucaparib[tiab] OR Rubraca[tiab] OR niraparib[tiab] OR Zejula[tiab] OR BRAF/MEK inhibitor*[tiab] OR BRAF MEK inhibitor*[tiab] OR encorafenib[tiab] OR Braftovi[tiab] OR binimetinib[tiab] OR Mektovi[tiab] OR vemurafenib[tiab] OR Zelboraf[tiab] OR cobimetinib[tiab] OR Cotellic[tiab] OR dabrafenib[tiab] OR Tafinlar[tiab] OR trametinib[tiab] OR Mekinist[tiab] OR EML4/ALK inhibitor*[tiab] OR EML4 ALK inhibitor*[tiab] OR alectinib[tiab] OR Alecensa[tiab] OR ceritinib[tiab] OR Zykadia[tiab] OR crizotinib[tiab] OR Xalkori[tiab] OR brigatinib[tiab] OR Alunbrig[tiab] OR CDK 4/6 inhibitor*[tiab] OR cyclin-dependent kinase 4/6 inhibitor*[tiab] OR abemaciclib[tiab] OR Verzenio[tiab] OR palbociclib[tiab] OR Ibrance[tiab] OR ribociclib[tiab] OR Kisqali[tiab] OR c-MET inhibitor*[tiab] OR cabozantinib[tiab] OR Cometriq[tiab] OR BTK inhibitor*[tiab] OR Bruton's tyrosine kinase inhibitor*[tiab] OR Brutons tyrosine kinase inhibitor*[tiab] OR ibrutinib[tiab] OR Imbruvica[tiab])

Filters activated: publication date from 2013/01/01 to 2018/12/31.

\section{Ovid embase}

1. exp skin disease/

2. exp skin manifestation/

3. exp skin toxicity/

4. exp skin injury/

5. exp drug eruption/

6. exp skin defect/

7. exp rash/

8. exp hyperpigmentation/

9. exp melanosis/

10. exp hand foot syndrome/

11. exp hypersensitivity/

12. exp allergic reaction/ 
13. exp nail disease/

14. exp nail dystrophy/

15. exp petechia/

16. exp purpura/

17. exp photosensitivity/

18. exp photodermatosis/

19. exp pruritus/

20. exp urticaria/

21. exp stomatitis/

22. exp erythema multiforme/

23. exp dry skin/

24. exp skin lupus erythematosus/

25. exp dermatitis/

26. exp erythema/

27. exp Stevens Johnson syndrome/

28. (cutaneous reaction* or cutaneous toxicit* or cutaneous complication* or cutaneous drug eruption* or cutaneous adverse

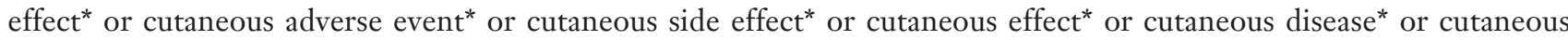
manifestation* or cutaneous injur* or cutaneous abnormalit* or dermatologic reaction* or dermatologic toxicit* or dermatologic complication* or dermatologic drug eruption* or dermatologic adverse effect* or dermatologic adverse event* or dermatologic side effect* or dermatologic effect* or dermatologic disease* or dermatologic manifestation* or dermatologic injur* or dermatologic abnormalit ${ }^{*}$ or skin reaction* ${ }^{*}$ or skin toxicit* or skin complication* or skin eruption* or skin side effect* or skin effect* or skin manifestation* or skin injur* or skin abnormalit* or skin damage or rash* or exanthema or hyperpigmentation or hypermelanos* or hand-foot syndrome or palmar-plantar erythrodysesthesia or palmoplantar erythrodysesthesia or hypersensitivit* or allergic reaction* or mucocutaneous or nail dystroph* or nail disease* or nail disorder* or nail toxicit* or nail change* or petechiae or purpura or photosensiti* or photodermatitis or pruritus or urticaria or stomatit* or erythema multiforme or dry skin or cutaneous lupus erythematosus or dermatitis or dermatos* or erythema or stevens-johnson syndrome).ti,ab.

29. or/1-28

30. exp nicotinamide adenine dinucleotide adenosine diphosphate ribosyltransferase inhibitor/

31. exp talazoparib/

32. exp olaparib/

33. exp rucaparib/

34. exp niraparib/

35. exp encorafenib/

36. exp binimetinib/

37. exp vemurafenib/

38. exp cobimetinib/

39. exp dabrafenib/

40. exp trametinib/

41. exp alectinib/

42. exp ceritinib/

43. exp crizotinib/

44. exp brigatinib/

45. exp abemaciclib/

46. exp palbociclib/

47. exp ribociclib/

48. exp cabozantinib/

49. exp Bruton tyrosine kinase inhibitor/ 
50. exp ibrutinib/

51. (PARP inhibitor* or polyadenosine ribose polymerase inhibitor* or poly ADP ribose polymerase inhibitor* or poly ADP-ribosylation inhibitor* or talazoparib or Talzenna or olaparib or Lynparza or rucaparib or Rubraca or niraparib or Zejula or BRAF MEK inhibitor* or encorafenib or Braftovi or binimetinib or Mektovi or vemurafenib or Zelboraf or cobimetinib or Cotellic or dabrafenib or Tafinlar or trametinib or Mekinist).ti,ab.

52. (EML4 ALK inhibitor* or alectinib or Alecensa or ceritinib or Zykadia or crizotinib or Xalkori or brigatinib or Alunbrig or CDK 46 inhibitor* or cyclin-dependent kinase 46 inhibitor* or abemaciclib or Verzenio or palbociclib or Ibrance or ribociclib or Kisqali or c-MET inhibitor* or cabozantinib or Cometriq).ti,ab.

53. (BTK inhibitor* or Bruton tyrosine kinase inhibitor* or Brutons tyrosine kinase inhibitor* or ibrutinib or Imbruvica). ti,ab.

54. or $/ 30-53$

55. 29 and 54

56. limit 55 to $\mathrm{yr}=" 2013$-Current"

\section{Ovid international pharmaceutical abstracts}

1. (cutaneous reaction* or cutaneous toxicit* or cutaneous complication* or cutaneous drug eruption* or cutaneous adverse effect* or cutaneous adverse event* or cutaneous side effect* or cutaneous effect* or cutaneous disease* or cutaneous manifestation* or cutaneous injur* or cutaneous abnormalit* or dermatologic reaction* or dermatologic toxicit* or dermatologic complication* or dermatologic drug eruption* or dermatologic adverse effect* or dermatologic adverse event $^{*}$ or dermatologic side effect* ${ }^{*}$ dermatologic effect* ${ }^{*}$ dermatologic disease* ${ }^{*}$ dermatologic manifestation* or dermatologic injur* or dermatologic abnormalit* or skin reaction* or skin toxicit* or skin complication* or skin eruption* or skin side effect* or skin effect* ${ }^{*}$ skin manifestation* ${ }^{*}$ or skin injur* or skin abnormalit* or skin damage or rash* or exanthema or hyperpigmentation or hypermelanos* or hand-foot syndrome or palmar-plantar erythrodysesthesia or palmoplantar erythrodysesthesia or hypersensitivit* or allergic reaction* or mucocutaneous or nail dystroph* or nail disease* $^{*}$ or nail disorder* or nail toxicit* or nail change* or petechiae or purpura or photosensiti* or photodermatitis or pruritus or urticaria or stomatit* or erythema multiforme or dry skin or cutaneous lupus erythematosus or dermatitis or dermatos* or erythema or stevens-johnson syndrome).ti,ab.

2. (PARP inhibitor* or polyadenosine ribose polymerase inhibitor* or poly ADP ribose polymerase inhibitor* or poly ADP-ribosylation inhibitor* or talazoparib or Talzenna or olaparib or Lynparza or rucaparib or Rubraca or niraparib or Zejula or BRAF MEK inhibitor* or encorafenib or Braftovi or binimetinib or Mektovi or vemurafenib or Zelboraf or cobimetinib or Cotellic or dabrafenib or Tafinlar or trametinib or Mekinist).ti,ab.

3. (EML4 ALK inhibitor* or alectinib or Alecensa or ceritinib or Zykadia or crizotinib or Xalkori or brigatinib or Alunbrig or CDK 46 inhibitor* or cyclin-dependent kinase 46 inhibitor* or abemaciclib or Verzenio or palbociclib or Ibrance or ribociclib or Kisqali or c-MET inhibitor* or cabozantinib or Cometriq).ti,ab.

4. (BTK inhibitor* or Bruton tyrosine kinase inhibitor* or Brutons tyrosine kinase inhibitor* or ibrutinib or Imbruvica). ti,ab.

5. or $/ 2-4$

6. 1 and 5

7. limit 6 to $\mathrm{yr}=" 2013$-Current"

\section{Additional search strategies: idelalisib/copanlisib}

The following search terms were entered into PubMed(MEDLINE): (("idelalisib"[Supplementary Concept] OR "idelalisib"[All Fields]) OR ("2-amino-N-(7-methoxy-8-(3-morpholinopropoxy)-2,3-dihydroimidazo(1,2-c)quinazolin4-yl)pyrimidine-5-carboxamide"[Supplementary Concept] OR "2-amino-N-(7-methoxy-8-(3-morpholinopropoxy)2,3-dihydroimidazo(1,2-c)quinazolin-4-yl)pyrimidine-5-carboxamide"[All Fields] OR "copanlisib"[All Fields]) OR ("idelalisib"[Supplementary Concept] OR "idelalisib"[All Fields] OR "zydelig"[All Fields]) OR Aliqopa[All Fields]) 
AND ((("skin"[MeSH Terms] OR "skin"[All Fields]) AND ("toxicity"[Subheading] OR "toxicity"[All Fields])) OR ("skin manifestations"[MeSH Terms] OR ("skin"[All Fields] AND "manifestations"[All Fields]) OR "skin manifestations"[All Fields]) OR ("exanthema"[MeSH Terms] OR "exanthema"[All Fields] OR "rash"[All Fields]) OR (cutaneous[All Fields] AND ("toxicity"[Subheading] OR "toxicity"[All Fields])) OR (cutaneous[All Fields] AND manifestations[All Fields]) OR ("handfoot syndrome"[MeSH Terms] OR ("hand-foot"[All Fields] AND "syndrome"[All Fields]) OR "hand-foot syndrome"[All Fields] OR ("palmar"[All Fields] AND "plantar"[All Fields] AND "erythrodysesthesia"[All Fields]) OR "palmar plantar erythrodysesthesia"[All Fields]) OR ("stevens-johnson syndrome"[MeSH Terms] OR ("stevens-johnson"[All Fields] AND "syndrome"[All Fields]) OR "stevens-johnson syndrome"[All Fields] OR ("stevens"[All Fields] AND "johnson"[All Fields] AND "syndrome"[All Fields]) OR "stevens johnson syndrome"[All Fields]) OR ("stevens-johnson syndrome"[MeSH Terms] OR ("stevens-johnson"[All Fields] AND "syndrome"[All Fields]) OR "stevens-johnson syndrome"[All Fields] OR ("toxic"[All Fields] AND "epidermal"[All Fields] AND "necrolysis"[All Fields]) OR "toxic epidermal necrolysis"[All Fields]) OR ("toxicity"[Subheading] OR "toxicity"[All Fields]))

Seventy-two results were obtained, of which 4 were eventually used in the review.

\section{Regorafenib}

The following search terms were entered into PubMed(MEDLINE): (("regorafenib"[Supplementary Concept] OR "regorafenib"[All Fields]) OR "Stivarga"[All Fields]) AND ((("skin"[MeSH Terms] OR "skin"[All Fields]) AND ("toxicity"[Subheading] OR "toxicity"[All Fields])) OR ("skin manifestations"[MeSH Terms] OR ("skin"[All Fields] AND "manifestations"[All Fields]) OR "skin manifestations"[All Fields]) OR ("exanthema"[MeSH Terms] OR "exanthema"[All Fields] OR "rash"[All Fields]) OR (cutaneous[All Fields] AND ("toxicity"[Subheading] OR "toxicity"[All Fields])) OR (cutaneous[All Fields] AND manifestations[All Fields]) OR ("hand-foot syndrome"[MeSH Terms] OR ("hand-foot"[All Fields] AND "syndrome"[All Fields]) OR "hand-foot syndrome"[All Fields] OR ("palmar"[All Fields] AND "plantar"[All Fields] AND "erythrodysesthesia"[All Fields]) OR "palmar plantar erythrodysesthesia"[All Fields]) OR ("stevensjohnson syndrome"[MeSH Terms] OR ("stevens-johnson"[All Fields] AND "syndrome"[All Fields]) OR "stevens-johnson syndrome"[All Fields] OR ("stevens"[All Fields] AND "johnson"[All Fields] AND "syndrome"[All Fields]) OR "stevens johnson syndrome"[All Fields]) OR ("stevens-johnson syndrome"[MeSH Terms] OR ("stevens-johnson"[All Fields] AND "syndrome"[All Fields]) OR "stevens-johnson syndrome"[All Fields] OR ("toxic"[All Fields] AND "epidermal"[All Fields] AND "necrolysis"[All Fields]) OR "toxic epidermal necrolysis"[All Fields]) OR ("toxicity"[Subheading] OR "toxicity"[All Fields]))

One hundred sixty results were obtained, of which were eventually used in the review. 